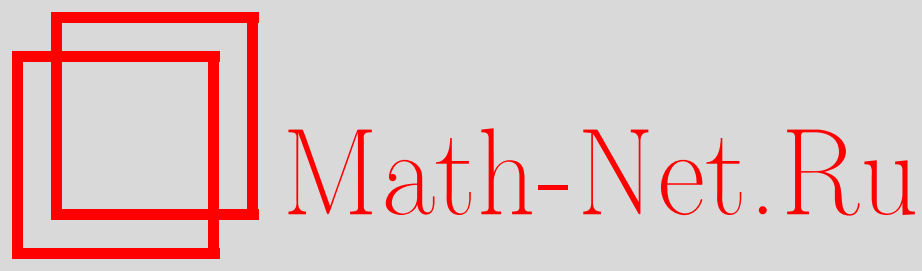

А. В. Киселев, Й. В. ван де Лёр, Алгебры симметрий лагранжевых систем лиувиллева типа, ТМФ, 2010, том 162, номер 2, 179-195

DOI: https://doi.org/10.4213/tmf6463

Использование Общероссийского математического портала Math-Net.Ru подразумевает, что вы прочитали и согласны с пользовательским соглашением http://www . mathnet.ru/rus/agreement

Параметры загрузки:

IP : 54.210 .77 .194

26 апреля 2023 г., 12:24:36

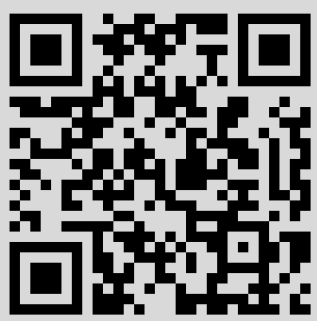




\section{АЛГЕБРЫ СИММЕТРИЙ ЛАГРАНЖЕВЫХ СИСТЕМ ЛИУВИЛЛЕВА ТИПА}

Явно вычислены образующие и коммутационные соотношения в алгебрах высших симметрий класса гиперболических лагранжевых систем лиувиллева типа, в частности для двумерных цепочек Тоды, связанных с полупростыми комплексными алгебрами Ли.

Ключевые слова: симметрии, двумерные цепочки Тоды, системы лиувиллева типа, гамильтоновы иерархии, скобки.

\section{1. ВВЕДЕНИЕ}

В работе приведено описание образующих и соотношений в алгебрах высших симметрий для класса лагранжевых гиперболических систем лиувиллева типа, интегрируемых по Дарбу [1]-[3]. Существует много неэквивалентных определений таких уравнений с частными производными [1], [3], [4]; мы исследуем системы $\mathcal{E}_{\mathrm{L}}$, допускающие столько же первых интегралов у каждого из характеристических уравнений $D_{y}(w) \doteq 0$ и $D_{x}(\bar{w}) \doteq 0$ на $\mathcal{E}_{\mathrm{L}}$, сколько имеется неизвестных функций. Двумерные цепочки Тоды $\boldsymbol{u}_{x y}=e^{K \boldsymbol{u}}$, связанные с полупростыми комплексными алгебрами Ли, - наиболее хорошо изученный пример таких уравнений [1], [2], [5]-[7]. Известно, что системы, принадлежащие рассматриваемому классу, допускают высшие симметрии вида $\varphi=\square(\phi)$, зависящие от свободных функциональных параметров $\phi={ }^{t}\left(\phi_{1}(x,[w]), \ldots, \phi_{r}(x,[w])\right)$ и принадлежащие образу линейных матричных дифференциальных операторов $\square$ в полных производных [6], [8]-[10]. Существование таких операторов $\square$ для систем лиувиллева типа было замечено в работах [1], [6] и [3], [10], в которых была обнаружена значимость линеаризаций $\ell_{w}^{(u)}$ первых интегралов $w$ для построения операторов $\square$. В статье [9] доказано, что при дополнительном предположении о том, что уравнение $\mathcal{E}_{\mathrm{L}}$ лагранжево, известный результат можно усилить и явно получить операторы $\square$ (см. ниже формулу (3)).

В настоящей работе выведены правила преобразования операторов $\square$ относительно не связанных между собой замен координат на их областях определения и в образах. Показано, что при естественных предположениях о геометрии уравнения $\mathcal{E}_{\mathrm{L}}$

${ }^{*}$ Mathematical Institute, University of Utrecht, Utrecht, The Netherlands.

E-mail: A.V.Kiselev@uu.nl, J.W.vandeLeur@uu.nl 
образы этих операторов замкнуты относительно коммутирования; следовательно, на областях определения операторов $\square$ также заданы структуры алгебры Ли. Возникающие при этом скобки мы вычисляем явно, что поставляет, при соответствующем индуцированном отображении структуры алгебры Ли, коммутационные соотношения в алгебрах симметрий $\operatorname{sym} \mathcal{E}_{\mathrm{L}}$. Для этой цели мы вводим вспомогательные гамильтоновы операторы с теми же областями определения, что у операторов $\square$.

ЗАмечАние 1 . Мы не предполагаем наличия у $\mathcal{E}_{\mathrm{L}}$ симметрии $x \leftrightarrow y$. Всюду мы рассматриваем $x$-компоненту алгебры $\operatorname{sym} \mathcal{E}_{\mathrm{L}}$, связанную с первыми интегралами $\left.w^{i} \in \operatorname{ker} D_{y}\right|_{\mathcal{E}_{\mathrm{L}}}$. Рассуждения остаются в силе и для соответствующей $y$-компоненты алгебры $\operatorname{sym} \mathcal{E}_{\mathrm{L}}$, причем две эти подалгебры коммутируют между собой. Для рассматриваемых нами лагранжевых систем $\mathcal{E}_{\mathrm{L}}$ интегралы $\left.\bar{w}^{\bar{\imath}} \in \operatorname{ker} D_{x}\right|_{\mathcal{E}_{\mathrm{L}}}$ в доказательствах не используются, в отличие от работы [10], в которой системы лиувиллева типа произвольные.

Полный список предположений о системах $\mathcal{E}_{\mathrm{L}}$ и их интегралах сформулирован в основной теореме 2 (см. также замечание 4). Отметим, впрочем, что рассуждения в разделе 2 остаются справедливыми и при менее ограничительных условиях. В частности, количество первых интегралов $w^{1}, \ldots, w^{r}$ характеристического уравнения, выполненного в силу $\mathcal{E}_{\mathrm{L}}$, может быть меньше, чем число неизвестных $u^{1}, \ldots, u^{m}$ в $\mathcal{E}_{\mathrm{L}}$. В этом случае вспомогательные матричные операторы $\hat{A}_{k}$ размера $r \times r(\mathrm{~cm}$. ниже формулу (7)) становятся меньше по размеру, но остаются гамильтоновыми (пример - вторая пуассонова структура для уравнения Кортевега-де Фриза (КдФ), построенная из двумерной цепочки Тоды с единственным интегралом, - приведен в работе [9]).

Структура статьи следующая. В разделе 2 мы определяем операторы $\square$, с помощью которых строятся образующие алгебры симметрий систем $\mathcal{E}_{\mathrm{L}}$, и вводим вспомогательные гамильтоновы операторы. При этом мы заново получаем высшие пуассоновы структуры иерархий Дринфельда-Соколова [11] на двумерных цепочках Тоды, ассоциированных с полупростыми комплексными алгебрами Ли; для цепочки Тоды, заданной по алгебре $\mathrm{A}_{2}$, приведен пример. В разделе 3 установлена замкнутость образов операторов $\square$ относительно коммутирования и вычислены структурные соотношения в алгебрах $\operatorname{sym} \mathcal{E}_{\mathrm{L}}$; в качестве иллюстрации приведено уравнение Каупа-Буссинеска. Наконец, в разделе 4 обсуждаются некоторые свойства операторов, задающих симметрии систем лиувиллева типа, но не обязательно лагранжевых.

Все используемые понятия и конструкции из геометрии дифференциальных уравнений с частными производными стандартны (см. [12]-[14]). Мы придерживаемся обозначений из работ [9], [15], [16]. В настоящей статье развиваются идеи, сформулированные в работе [9].

\section{2. ОБРАЗУЮЩИЕ АЛГЕБРЫ СИММЕТРИЙ УРАВНЕНИЯ $\mathcal{E}_{\mathrm{L}}$}

ОПРЕДЕЛЕНИЕ. Система $\mathcal{E}=\left\{\boldsymbol{u}_{x y}=f\left(\boldsymbol{u}, \boldsymbol{u}_{x}, \boldsymbol{u}_{y} ; x, y\right)\right\}$, состоящая из $m$ гиперболических уравнений относительно неизвестных $\boldsymbol{u}=\left(u^{1}, \ldots, u^{m}\right)$, является системой лиувиллева типа, если существуют нетривиальные первые интегралы

$$
w^{1}, \ldots,\left.w^{r} \in \operatorname{ker} D_{y}\right|_{\mathcal{E}} ; \quad \bar{w}^{1}, \ldots,\left.\bar{w}^{\bar{r}} \in \operatorname{ker} D_{x}\right|_{\mathcal{E}}, \quad 0<r, \bar{r} \leqslant m,
$$


линейных характеристических уравнений первого порядка $\left.D_{y}\right|_{\mathcal{E}}\left(w^{i}\right) \doteq 0$ и $\left.D_{x}\right|_{\mathcal{E}}\left(\bar{w}^{\bar{\jmath}}\right) \doteq$ 0, выполненных в силу $(\doteq)$ уравнения $\mathcal{E}$.

Пример 1. В работе [2] было показано, что двумерные цепочки Тоды [5] $u_{x y}^{i}=$ $e^{K_{j}^{i} u^{j}}$, ассоциированные с полупростыми комплексными алгебрами Ли с матрицами Картана $K$, допускают максимальные $(r=\bar{r}=m)$ наборы интегралов. Различные методы построения интегралов $w^{i}, \bar{w}^{\bar{j}}$ для экспоненциально нелинейных цепочек Тоды были предложены в статьях [3], [4], [7]. После сдвига на -1 дифференциальные порядки (по $\boldsymbol{u}$ ) интегралов $w^{1}, \ldots, w^{r}$ равны показателям соответствующих полупростых комплексных алгебр Ли ранга $r$ (см. с. 21 в [2]).

Например, ниже мы рассматриваем лагранжеву двумерную систему Тоды $\mathcal{E}_{\text {Tода }}$ ассоциированную с простой алгеброй Ли $\mathfrak{s l}_{3}(\mathbb{C})$ (см. [1], [5], [7]):

$$
\mathcal{E}_{\text {Toda }}=\left\{u_{x y}=e^{2 u-v}, \quad v_{x y}=e^{-u+2 v}, \quad K=\left(\begin{array}{cc}
2 & -1 \\
-1 & 2
\end{array}\right)\right\} .
$$

Интегралы (второго и третьего порядков) для системы (1) имеют вид соответственно (см., например, [17])

$$
\begin{aligned}
& w^{1}=u_{x x}+v_{x x}-u_{x}^{2}+u_{x} v_{x}-v_{x}^{2}, \\
& w^{2}=u_{x x x}-2 u_{x} u_{x x}+u_{x} v_{x x}+u_{x}^{2} v_{x}-u_{x} v_{x}^{2} .
\end{aligned}
$$

Производящие сечения $\varphi=\square(\phi(x,[w]))$ образующих алгебры высших симметрий уравнений лиувиллева типа задаются с помощью матричных дифференциальных операторов $\square$ в полных производных (см. [3], [6]). В случае, если система лиувиллева типа лагранжева, т. е. $\mathcal{E}_{\mathrm{L}}=\{F \equiv \mathbf{E}(\mathcal{L})=0\}$ (см. [8], [9], [18]), существование некоторых факторизаций для по крайней мере части симметрий - закономерный факт, который легко проверить следующим образом. Для интегралов $w$ существует оператор $\nabla$ такой, что производная $D_{y}(w)=\nabla(F)$ обращается в нуль на дифференциальном идеале $\{F=0\}^{\infty}$. В самом деле, производящее сечение $\psi_{I}=\left[\nabla^{*} \circ\left(\ell_{w}^{(u)}\right)^{*} \circ\left(\ell_{I}^{(w)}\right)^{*}\right](1)$ закона сохранения $\int I d x$ удовлетворяет уравнению $\ell_{\mathbf{E}(\mathcal{L})}^{*}\left(\psi_{I}\right) \doteq 0$ в силу $\mathcal{E}_{\mathrm{L}}$ при любой функции $I(x,[w])$ (см. [12]-[14]). Из условия Гельмгольца $\ell_{\mathbf{E}(\mathcal{L})}=\ell_{\mathbf{E}(\mathcal{L})}^{*}$ для линеаризации (производной Фреше) следует, что вектор

$$
\varphi[\phi]=\left.\left[\nabla^{*} \circ\left(\ell_{w}^{(u)}\right)^{*}\right](\phi(x,[w])) \in \operatorname{ker} \ell_{\mathbf{E}(\mathcal{L})}\right|_{\mathcal{E}_{\mathrm{L}}}
$$

является симметрией уравнения $\mathcal{E}_{\mathrm{L}}$ для всякого сечения $\phi=\left(\ell_{I}^{(w)}\right)^{*}(1)=\mathbf{E}_{w}(I d x)$. Стандартное гомологическое рассуждение (см. гл. 5 в [13] или $§ 7.8$ в [14]) показывает, что формула (2) задает симметрии лагранжевой системы $\mathcal{E}_{\mathrm{L}}$, даже если сечения $\phi$ не принадлежат образу вариационной производной $\mathbf{E}_{w}$ по $w$.

В этом разделе мы напомним процедуру построения операторов $\square$, задающих симметрии класса лагранжевых систем лиувиллева типа. Всюду предполагается, что интегралы $w$ минимальны, т. е. из $\left.I \in \operatorname{ker} D_{y}\right|_{\mathcal{E}_{\mathrm{L}}}$ следует $I=I(x,[w])$.

УтВЕРЖДЕНИЕ 1 [9]. Пусть $\kappa$-обратимая симметричная постоянная вещественная матрица размера $m \times m$. Далее, пусть $\mathcal{L}=\int L d x d y$ с плотностью

$$
L=-\frac{1}{2} \sum_{i, j} \kappa_{i j} u_{x}^{i} u_{y}^{j}-H_{L}(u ; x, y)
$$


- лагранжиан уравнения лиувиллева типа $\mathcal{E}_{\mathrm{L}}=\{\mathbf{E}(\mathcal{L})=0\}$. Введем канонические импульсъ $\mathfrak{m}=\partial L / \partial u_{y}$ и предположим, ито $w[\mathfrak{m}]=\left(w^{1}, \ldots, w^{r}\right)-$ набор минимальных интегралов уравнения $\mathcal{E}_{\mathrm{L}}$, принадлежащих ядру $\left.D_{y}\right|_{\mathcal{E}_{\mathrm{L}}}$. Тогда оператор

$$
\square=\left(\ell_{w}^{(\mathfrak{m})}\right)^{*},
$$

сопряженный $\kappa$ линеаризации интегралов по импульсам, задает нётеровы симметрии $\varphi_{\mathcal{L}}$ уравнения $\mathcal{E}_{\mathrm{L}}$ формулой

$$
\varphi_{\mathcal{L}}=\square\left(\frac{\delta \mathcal{H}}{\delta w}\right) \quad \forall \mathcal{H}=\int H(x,[w]) d x .
$$

СлеДСТвИЕ. В предположениях и обозначениях утверждения 1 сечение

$$
\varphi=\square(\phi(x,[w]))
$$

является симметрией уравнения лиувиллева типа $\mathcal{E}_{\mathrm{L}}$ для любого г-компонентного столби, $\phi={ }^{t}\left(\phi_{1}, \ldots, \phi_{r}\right)$.

ДоказАтельство. Рассмотрим расслоение струй $J^{\infty}(\xi)$ над расслоением $\xi: \mathbb{R}^{r} \times$ $\mathbb{R} \rightarrow \mathbb{R}$ с базой $\mathbb{R} \ni x$ и слоем $\mathbb{R}^{r}$, координатами на котором служат $w^{1}, \ldots, w^{r}$. Согласно утверждению 1 следствие справедливо для любого сечения $\phi$, лежащего в образе вариационной производной $\mathbf{E}_{w}$. Очевидно, ее образ содержит все вариационные ковекторы $\phi$ с компонентами $\phi_{i}(x) \in C^{\infty}(\mathbb{R})$ - функциями на базе нового расслоения $\xi$. Продолжение подстановки $w=w[\mathfrak{m}[u]]: J^{\infty}(\pi) \rightarrow \Gamma(\xi)$ преобразует компоненты сечений $\phi$ в гладкие дифференциальные функции от $u$ (одной буквой мы обозначаем все множество из $m$ координат в слое расслоения $\pi$ над той же самой базой $\mathbb{R} \ni x)$. Вспомним теперь, что $\square$ - оператор в полных производных $D_{x}$, действие которых на дифференциальные функции $f[u]$ определено формулой

$$
j_{\infty}(s)\left(D_{x}(f)\right):=\frac{\partial}{\partial x}\left(j_{\infty}(s)(f)\right),
$$

т. е. через ограничение $j_{\infty}(s)(f)$ функции $f$ на струи $j_{\infty}(s)$ сечений $u=s(x)$. Мы получаем, следовательно, $\phi_{i}(x)=\phi_{i}(x,[w[\mathfrak{m}[s(x)]]])$, откуда и следует доказываемое утверждение.

ЗАмЕчАниЕ 2. Приведенное выше доказательство сочетает взятие вариационных производных по $w$ на одном пространстве струй с вычислением полных производных дифференциальных функций от $u$ на другом пространстве струй над той же самой базой. Если эти два пространства совпадают, рассуждения сводятся к определению производной $D_{x}$. В таком случае схема доказательства называется приниипом подстановки ([13], см. подробное обсуждение в [14]).

Теорема 1. При дифференииальных заменах $\widetilde{w}=\widetilde{w}[w]$ u $\tilde{u}=\tilde{u}[u]$ координат $w^{1}, \ldots, w^{r} u u^{1}, \ldots, u^{m}$ в расслоениях бесконечных струй над $\xi u \pi$, соответствующих области определения и образу оператора $\square$, этот оператор преобразуется по бормуле

$$
\square \mapsto \tilde{\square}=\left.\ell_{\tilde{u}}^{(u)} \circ \square \circ\left(\ell_{\widetilde{w}}^{(w)}\right)^{*}\right|_{\substack{w=w[u] \\ u=u[\tilde{u}]}} .
$$


ДоказАтЕЛЬство. Преобразование скоростей $\tilde{\varphi}=\ell_{\tilde{u}}^{(u)}(\varphi)$ очевидно. При дифференциальных заменах $w=w[\widetilde{w}]$ интегралов сечения $\phi=\delta \mathcal{H} / \delta w$ преобразуются по формуле $\phi=\left(\ell_{\widetilde{w}}^{(w)}\right)^{*}(\tilde{\phi})$, что позволяет корректно определить оператор $\square$ на всем образе $\operatorname{im} \mathbf{E}_{w}$. Именно, оператор отображает вариационные ковекторы для расслоения $\xi$ в эволюционные дифференцирования на пространстве струй над другим расслоением $\pi$. Повторяя рассуждение, использованное при доказательстве следствия утверждения 1 , получаем правило (6) преобразования оператора $\square$ на всей области определения.

В теореме 1 мы показали, что сечения из области определения оператора $\square$ преобразуются по тому же правилу, что и аргументы гамильтоновых операторов. В самом деле, тому имеются глубокие причины.

Интегралы $w[\mathfrak{m}]$ лагранжевых систем $\mathcal{E}_{\mathrm{L}}$ лиувиллева типа порождают подстановки Миуры из коммутативных гамильтоновых иерархий $\mathfrak{B}$ (того же типа, что и модифицированное уравнение $\mathrm{Kд} \Phi)$ нётеровых симметрий уравнения $\mathcal{E}_{\mathrm{L}}$ во вполне интегрируемые иерархии $\mathfrak{A}$ типа КдФ высших симметрий многокомпонентных волновых уравнений $\mathcal{E}_{\varnothing}=\left\{s_{x y}=0\right\}$ (см. ниже). Естественным примером служит потенциальное модифицированное уравнение КдФ

$$
u_{t}=-\frac{1}{2} u_{x x x}+u_{x}^{3}
$$

которое преобразуется в уравнение КдФ

$$
w_{t}=-\frac{1}{2} w_{x x x}+3 w w_{x}
$$

посредством замены $w=u_{x}^{2}-u_{x x}$. Этот пример был детально изучен в работе [9]. Такой метод порождения полезных дифференциальных подстановок интегралами $w$ систем лиувиллева типа был открыт в работе [19] (см. обсуждение в [3]). Данный факт был использован в работе [20] для классификации дифференциальных подстановок первого порядка.

Гамильтонианы $\mathcal{H}_{i}[\mathfrak{m}]=\mathcal{H}_{i}[w[\mathfrak{m}]]$ иерархий $\mathfrak{A}$ и $\mathfrak{B}$ общие, они связаны подстановкой Миуры $w[\mathfrak{m}]$. Гамильтоновы структуры в схемах Магри для иерархий $\mathfrak{A}$ и $\mathfrak{B}$ согласованы операторами $\square$, которые отображают “косимметрии” $\phi_{i}$ иерархии $\mathfrak{A}$ в симметрии $\varphi_{i+1}$ модифицированной иерархии $\mathfrak{B}$. Подчеркнем, что первый гамильтонов оператор $\widehat{B}_{1}=\left(\ell_{\mathfrak{m}}^{(u)}\right)^{*}$ иерархии $\mathfrak{B}$ происходит из дифференциальной связи $\mathfrak{m}=$ $\partial L / \partial u_{y}$ между каноническими координатами $u$ и импульсами $\mathfrak{m}$ для уравнения $\mathcal{E}_{\mathrm{L}}$. Используя явно заданный "младший" оператор $\widehat{B}_{1}$ и дифференциально-функциональную замкнутость (относительно $[w]$ ) скоростей эволюции интегралов вдоль симметрий (см. [3]), мы воспроизводим классическую схему задания высших пуассоновых структур подстановками Миуры [21].

ЛЕмма 1. Рассмотрим линейный дифференциалъный оператор

$$
\hat{A}_{k}=\square^{*} \circ \widehat{B}_{1} \circ \square
$$


отображающий вариационные ковекторы для расслоения струй $J^{\infty}(\xi)$ над $\xi$ в эволющионные векторные поля на нем, т.е.

$$
\hat{A}_{k}: \Gamma(\hat{\xi}) \bigotimes_{C^{\infty}(\mathbb{R})} C^{\infty}\left(J^{\infty}(\xi)\right) \rightarrow \Gamma(\xi) \bigotimes_{C^{\infty}(\mathbb{R})} C^{\infty}\left(J^{\infty}(\xi)\right) .
$$

Тогда оператор (7) гамильтонов и определяет пуассонову структуру для иерар-

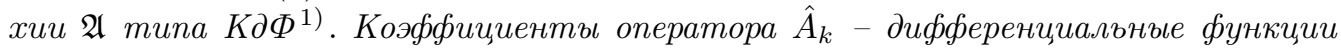
om $w$.

ДокАЗАТЕЛЬСтво. По построению скобка Пуассона

$$
\left\{\mathcal{H}_{1}, \mathcal{H}_{2}\right\}_{\hat{A}_{k}}=\left\langle\mathbf{E}_{w}\left(\mathcal{H}_{1}\right), \hat{A}_{k}\left(\mathbf{E}_{w}\left(\mathcal{H}_{2}\right)\right)\right\rangle
$$

удовлетворяет равенству

$$
\left\{\mathcal{H}_{1}[w], \mathcal{H}_{2}[w]\right\}_{\hat{A}_{k}}=\left\{\mathcal{H}_{1}[w[\mathfrak{m}]], \mathcal{H}_{2}[w[\mathfrak{m}]]\right\} \widehat{B}_{1}
$$

Следовательно, левая часть формулы (8) билинейна, кососимметрична и удовлетворяет тождеству Якоби. Кроме того, она измеряет скорость эволюции интегралов $w$ вдоль нётеровой симметрии уравнения $\mathcal{E}_{\mathrm{L}}$. Поскольку эволюционные дифференцирования перестановочны с полной производной $D_{y}$, скорость $\left\{\mathcal{H}_{1}, \mathcal{H}_{2}\right\}_{\hat{A}_{k}}$ лежит в ядре $\left.\operatorname{ker} D_{y}\right|_{\mathcal{E}_{\mathrm{L}}}$, а значит, плотность полученного функционала - дифференциальная функция минимальных интегралов $w$.

Многокомпонентное волновое уравнение $\mathcal{E}_{\varnothing}=\left\{s_{x y}=0\right\}$, симметрии которого содержат иерархию $\mathfrak{A}$ и такое, что условие $\hat{A}_{1}=\left(\ell_{w}^{(s)}\right)^{*}$ задает дифференциальную связь между координатами $s$ и импульсами $w$ для уравнения $\mathcal{E}_{\varnothing}$, выбирается следующим образом. Нужно, чтобы первая структура $A_{1}=\hat{A}_{1}^{-1}$ для иерархии $\mathfrak{A}$ факторизовала высшую гамильтонову структуру для $\mathfrak{B}$. Таким образом, имеем $B_{k^{\prime}}=\square \circ A_{1} \circ \square^{*}$, где $k^{\prime}=k^{\prime}\left(\square,\left(\ell_{w}^{(s)}\right)^{*}\right) \geqslant 2$.

Пример 2. Рассмотрим двумерную лагранжеву систему Тоды (1). Плотность $L$ ее лагранжиана имеет вид

$$
L=-\frac{1}{2}\left(\left(2 u_{x}-v_{x}\right) u_{y}+\left(2 v_{x}-u_{x}\right) v_{y}\right)-e^{2 u-v}-e^{2 v-u}
$$

Соответственно определим импульсы формулами $\mathfrak{m}^{1}:=2 u_{x}-v_{x}, \mathfrak{m}^{2}:=2 v_{x}-u_{x}$, а затем выразим через них интегралы: $w=w[\mathfrak{m}]$. Все симметрии уравнения (1) имеют вид (с точностью до $x \leftrightarrow y) \varphi=\square\left(\phi\left(x,\left[w^{1}\right],\left[w^{2}\right]\right)\right)$, где $\phi={ }^{t}\left(\phi_{1}, \phi_{2}\right)-$ пара произвольных функций, $\square$ - матричный (размера $2 \times 2$ ) оператор в полных производных, который имеет вид

$$
\square=\ell_{w^{1}, w^{2}}^{\left(\mathfrak{m}^{1}, \mathfrak{m}^{2}\right)}=\left(\begin{array}{rr}
u_{x}+D_{x} & -\frac{2}{3} D_{x}^{2}-u_{x} D_{x}-\frac{1}{3} u_{x}^{2}-\frac{2}{3} u_{x} v_{x}+\frac{2}{3} v_{x}^{2}+\frac{1}{3} u_{x x}-\frac{2}{3} v_{x x} \\
v_{x}+D_{x} & -\frac{1}{3} D_{x}^{2}+\frac{2}{3} u_{x x}-\frac{1}{3} v_{x x}-\frac{2}{3} u_{x}^{2}+\frac{2}{3} u_{x} v_{x}+\frac{1}{3} v_{x}^{2}
\end{array}\right) .
$$

1)В большинстве случаев получается одна из высших структур для иерархии $\mathfrak{A}$, на что мы указываем наличием нижнего индекса $k=k(\square, \mathfrak{m}) \geqslant 2$. Выбор "младшего" оператора $\hat{A}_{1}$ для $\mathfrak{A}$ будет обсуждаться ниже. 
Компоненты возникающего гамильтонова оператора $\hat{A}_{2}=\left\|A_{i j}, 1 \leqslant i, j \leqslant 2\right\|$ получаются следующими [13]:

$$
\begin{aligned}
A_{11}= & 2 D_{x}^{3}+2 w^{1} D_{x}+w_{x}^{1} \\
A_{12}= & -D_{x}^{4}-w^{1} D_{x}^{2}+\left(3 w^{2}-2 w_{x}^{1}\right) D_{x}+\left(2 w_{x}^{2}-w_{x x}^{1}\right) \\
A_{21}= & D_{x}^{4}+w^{1} D_{x}^{2}+3 w^{2} D_{x}+w_{x}^{2} \\
A_{22}= & -\frac{2}{3} D_{x}^{5}-\frac{4}{3} w^{1} D_{x}^{3}-2 w_{x}^{1} D_{x}^{2}+\left(2 w_{x}^{2}-2 w_{x x}^{1}-\frac{2}{3}\left(w^{1}\right)^{2}\right) D_{x}+ \\
& \quad+\frac{1}{3}\left(3 w_{x x}^{2}-2 w_{x x x}^{1}-2 w^{1} w_{x}^{1}\right) .
\end{aligned}
$$

Сдвиг $w^{2} \mapsto w^{2}+\lambda$ второго интеграла порождает "младший” гамильтонов оператор 2$)$

$$
\hat{A}_{1}^{(2)}:=\left.\frac{d}{d \lambda}\right|_{\lambda=0}\left(\hat{A}_{2}\right)=\left(\begin{array}{cc}
0 & 3 D_{x} \\
3 D_{x} & 0
\end{array}\right),
$$

который согласован с $\hat{A}_{2}$.

Пара $\left(\hat{A}_{1}^{(2)}, \hat{A}_{2}\right)$ - это и есть бигамильтонова структура для уравнения Буссинеска

$$
w_{t}^{1}=2 w_{x}^{2}-w_{x x}^{1}, \quad w_{t}^{2}=-\frac{2}{3} w_{x x x}^{1}-\frac{2}{3} w^{1} w_{x}^{1}+w_{x x}^{2} .
$$

Симметрия $w_{x}=\left(\hat{A}_{2} \circ \delta / \delta w\right)\left(\int w^{1} d x\right)$ начинает вторую последовательность гамильтоновых потоков в иерархии $\mathfrak{A}$ уравнения Буссинеска. Модифицированная иерархия Буссинеска $\mathfrak{B}$ наследует обе последовательности гамильтонианов от $\mathfrak{A}$ при помощи подстановки Миуры $w=w[\mathfrak{m}]$, где учтено $\mathfrak{m}=\mathfrak{m}[u]$. Именно, для любого гамильтониана $\mathcal{H}[w]$ потоки $u_{\tau}=\delta \mathcal{H}[\mathfrak{m}] / \delta \mathfrak{m}, \mathfrak{m}_{\tau}=-\delta \mathcal{H}[\mathfrak{m}[u]] / \delta u$ принадлежат модифицированной иерархии $\mathfrak{B}$. Скорости $u_{\tau}$ образуют коммутативную подалгебру нётеровых симметрий двумерной цепочки Тоды (1).

\section{3. КОММУТАЦИОННЫЕ СООТНОШЕНИЯ В $\operatorname{sym} \mathcal{E}_{\mathrm{L}}$}

В этом разделе будет установлена замкнутость образов операторов (3) относительно коммутирования, доказательство базируется на хорошо известном аналогичном свойстве вспомогательных гамильтоновых операторов (7). Одновременно будут описаны коммутационные соотношения в алгебре симметрий вида $(5)$ уравнения $\mathcal{E}_{\mathrm{L}}$.

Для начала рассмотрим произвольный линейный оператор $A$ в полных производных, аргументами $\phi(x,[w])={ }^{t}\left(\phi_{1}, \ldots, \phi_{r}\right)$ которого служат вариационные ковекторы для расслоения бесконечных струй над $\xi$. Допустим, что образ оператора $A$ в алгебре Ли эволюционных векторных полей $\partial_{\varphi}$ замкнут относительно коммутирования: $[\operatorname{im} A, \operatorname{im} A] \subseteq \operatorname{im} A$. Согласно правилу Лейбница в скобке полей $A\left(\phi^{\prime}\right), A\left(\phi^{\prime \prime}\right)$, принадлежащих образу оператора $A$, появляются два вида слагаемых:

$$
\left[A\left(\phi^{\prime}\right), A\left(\phi^{\prime \prime}\right)\right]=A\left(\partial_{A\left(\phi^{\prime}\right)}\left(\phi^{\prime \prime}\right)-\partial_{A\left(\phi^{\prime \prime}\right)}\left(\phi^{\prime}\right)\right)+\left(\partial_{A\left(\phi^{\prime}\right)}(A)\left(\phi^{\prime \prime}\right)-\partial_{A\left(\phi^{\prime \prime}\right)}(A)\left(\phi^{\prime}\right)\right)
$$
вым.

2) Аналогичный ему оператор $\hat{A}_{1}^{(1)}=\left.\frac{d}{d \mu}\right|_{\mu=0}\left(\hat{A}_{k}\right)$, где $w^{1} \mapsto w^{1}+\mu$, оказывается негамильтоно- 
В первом слагаемом использована перестановочность эволюционных дифференцирований и полных производных. Второе слагаемое попадает в образ оператора $A$ по построению.

Коммутатор $\left.[\cdot, \cdot]\right|_{\text {im } A}$ индуцирует структуру алгебры Ли $[\cdot, \cdot]_{A}$ в факторе $\Omega\left(\xi_{\pi}\right)$ области определения оператора $A$ по его ядру:

$$
\left[A\left(\phi^{\prime}\right), A\left(\phi^{\prime \prime}\right)\right]=A\left(\left[\phi^{\prime}, \phi^{\prime \prime}\right]_{A}\right), \quad \phi^{\prime}, \phi^{\prime \prime} \in \Omega\left(\xi_{\pi}\right) .
$$

Эта скобка, определенная с точностью до $\operatorname{ker} A$, равняется

$$
\left[\phi^{\prime}, \phi^{\prime \prime}\right]_{A}=\partial_{A\left(\phi^{\prime}\right)}\left(\phi^{\prime \prime}\right)-\partial_{A\left(\phi^{\prime \prime}\right)}\left(\phi^{\prime}\right)+\left\{\left\{\phi^{\prime}, \phi^{\prime \prime}\right\}_{A} .\right.
$$

Она содержит два стандартных слагаемых и кососимметричную билинейную скобку $\{\{\cdot, \cdot\}\}_{A}$.

Лемма 2 [13], [14]. Образ гамильтонова оператора $\hat{A}=\left\|\sum_{\boldsymbol{\tau}} A_{\boldsymbol{\tau}}^{i j}(x,[w]) \cdot D_{\boldsymbol{\tau}}\right\|$ замкнут относительно коммутирования; $k$-я компонента, $1 \leqslant k \leqslant r$, возникающей на области определения оператора $\hat{A}$ скобки $\{\{\cdot, \cdot\}\}_{\hat{A}}$ вычисляется по формуле

$$
\left\{\left\{\phi^{\prime}, \phi^{\prime \prime}\right\}\right\}_{\hat{A}}^{k}=\sum_{|\boldsymbol{\sigma}| \geqslant 0} \sum_{i=1}^{r}(-1)^{|\boldsymbol{\sigma}|}\left(D_{\boldsymbol{\sigma}} \circ\left[\sum_{|\boldsymbol{\tau}| \geqslant 0} \sum_{j=1}^{r} D_{\boldsymbol{\tau}}\left(\phi_{j}^{\prime}\right) \cdot \frac{\partial A_{\boldsymbol{\tau}}^{i j}}{\partial w_{\boldsymbol{\sigma}}^{k}}\right]\right)\left(\phi_{i}^{\prime \prime}\right) .
$$

Коэфбициенты билинейнъх по $\phi^{\prime}, \phi^{\prime \prime}$ слагаемых в скобке $\{\{\cdot, \cdot\}\}_{\hat{A}}-$ дифберенциальные функиии переменных $w$.

Перейдем теперь от рассмотрения гамильтоновых операторов (7) к операторам $\square$, имеющим ту же самую область определения, что и $\hat{A}_{k}$, но со значениями в другой алгебре Ли. Наш основной результат состоит в следующем.

ТЕОРема 2. Пусть на открытом всюду плотном подмножестве лагранжевой системы $\mathcal{E}_{\mathrm{L}}=\left\{\boldsymbol{u}_{x y}=f(\boldsymbol{u} ; x, y)\right\}$ лиувиллева типа (см. утверждение 1$)$ выполнены следуюшие предположения ${ }^{3)}$ :

а) постоянная симметричная вещественная матрица $\kappa$ в кинетическом слагаемом плотности лагранжиана $\mathcal{L}$ обратима;

б) линеаризачия $\ell_{f}^{(u)}=\left\|\partial f^{i} / \partial u^{j}\right\| \equiv f^{\prime}(\boldsymbol{u} ; x, y)$ правой части уравнения $\mathcal{E}_{\mathrm{L}}-$ обратимая матрица;

в) имеется ровно столько интегралов $\left.w^{i}(x,[\mathfrak{m}]) \in \operatorname{ker} D_{y}\right|_{\mathcal{E}_{\mathrm{L}}}$, сколько в системе неизвестных $u^{j}$;

г) интеграль $w$ минимальны: из $\left.\Phi \in \operatorname{ker} D_{y}\right|_{\mathcal{E}_{\mathrm{L}}}$ следует $\Phi=(x,[w])$;

д) интеграль $w$ дифференциально-функционально независимьь ${ }^{4)}$ в том смысле, что $\Phi(x,[w[\mathfrak{m}]])=0$ означает $\Phi \equiv 0 ;$

е) матрица $\Lambda=\left\|\partial w^{i} / \partial \mathfrak{m}_{d(i)}^{j}\right\|$ размера $r \times m$, в которой $d(i):=\operatorname{ord}_{x} w^{i}-\partial u \oint \oint е-$ ренииальный порядок $i$-го интеграла $w^{i}[\mathfrak{m}]$, обратима (т.е. имеет максимальный ранг).

${ }^{3)}$ Список предположений может быть не минимальным, но в приведенной формулировке оказывается удобнее проверять каждое из них по отдельности.

4) Несуществование нетривиальной функции $\Phi$ и, следовательно, ее ненулевой линеаризации $\ell_{\Phi}^{(\mathfrak{m})}=\ell_{\Phi}^{(w)} \circ \ell_{w}^{(\mathfrak{m})}$ эквивалентно импликации $\left(\nabla \circ \ell_{w}^{(\mathfrak{m})}=0\right.$, где $\left.\nabla=\ell_{(\cdot)}^{(\mathfrak{m})}\right) \Rightarrow \nabla=0$. В этой форме требование невырожденности двойственно к (12) (см. ниже). 
Тогда верны следующие утверждения:

1) образ оператора (3) замкнут относительно коммутирования симметрий $\varphi=$ $\square(\phi(x,[w])) \in \operatorname{sym} \mathcal{E}_{\mathrm{L}} ;$

2) скобка $\{\{\cdot, \cdot\}\}_{\square}$, возникающая на области определения оператора $\square$, удовлетворяет равенству

$$
\left\{\left\{\phi^{\prime}, \phi^{\prime \prime}\right\}\right\}_{\square}=\left\{\left\{\phi^{\prime}, \phi^{\prime \prime}\right\}\right\}_{\hat{A}_{k}}, \quad \phi^{\prime}, \phi^{\prime \prime} \in \Omega\left(\xi_{\pi}\right) ;
$$

3) коэффициенты при билинейных слагаемых в скобке $\{\{\cdot, \cdot\}\}_{\square}-$ дифференциальные функции интегралов $w$.

ЗАмЕчАниЕ 3 . Из первого предположения теоремы следует, что система $\mathcal{E}_{\mathrm{L}}$ определена, нормальна и $\ell$-нормальна (см. раздел 4). Подчеркнем, что система $\mathcal{E}_{\mathrm{L}}-$ это по предположению и есть все множество уравнений, налагаемых на сечения $u=s(x, y) \in \Gamma(\pi)$.

Второе утверждение теоремы означает, что неоднозначность (по модулю $\mathrm{ker} \hat{A}_{k}$ ) выбора представителя из класса эквивалентности $\left\{\left\{\phi^{\prime}, \phi^{\prime \prime}\right\}\right\}_{\hat{A}_{k}}$ в правой части равенства (11) сводится к выбору элемента из ker $\square \subseteq \operatorname{ker} \hat{A}_{k}$. Мы докажем совпадение ядер для всех $\phi([w]) \in \Omega\left(\xi_{\pi}\right)$. Отсюда следует, что коммутационные соотношения в sym $\mathcal{E}_{\mathrm{L}}$, порожденные структурой алгебры Ли $(9 б)$ на $\Omega\left(\xi_{\pi}\right)$, удается выписать явно с помощью формулы (10) для $\hat{A}_{k}$.

Будучи следствием леммы 2 и первых двух утверждений теоремы 2 , третье утверждение является в то же время частным случаем утверждения 2 (см. ниже).

ДокАЗАТЕЛЬСТво тЕОРЕмы 2. Прежде всего заметим, что симметрии (5) не зависят ни от $u$, ни от $u_{y}, u_{y y}, \ldots$. Значит, это верно также и для коммутатора $\varphi=\left[\varphi^{\prime}, \varphi^{\prime \prime}\right] \in \operatorname{sym} \mathcal{E}_{\mathrm{L}}$ двух таких симметрий $\varphi^{\prime}=\square\left(\phi^{\prime}(x,[w])\right)$ и $\varphi^{\prime \prime}=\square\left(\phi^{\prime \prime}(x,[w])\right)$, поскольку скобка Ли - локальный бидифференциальный оператор.

Факторизация (7) и лемма 2 представимы в виде диаграммы

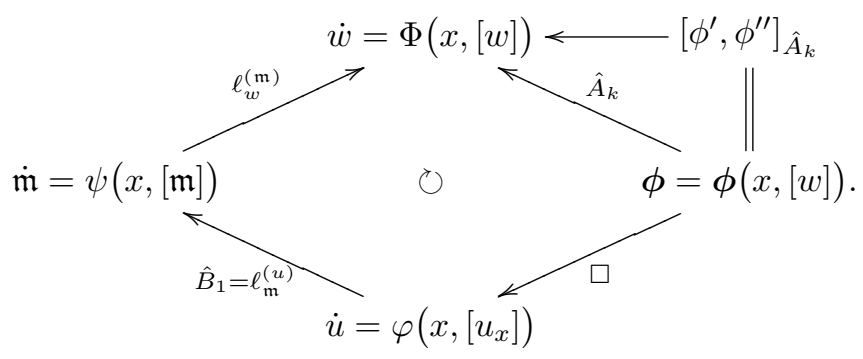

Коммутатор $\varphi=\left[\varphi^{\prime}, \varphi^{\prime \prime}\right]$ задает скорость $\Phi(x,[w])$ интегралов, равную ${ }^{5)} \partial_{\left[\varphi^{\prime}, \varphi^{\prime \prime}\right]}(w)=$ $\left[\hat{A}_{k}\left(\phi^{\prime}\right), \hat{A}_{k}\left(\phi^{\prime \prime}\right)\right]$. Так как образ гамильтонова оператора $\hat{A}_{k}$ замкнут относительно коммутирования, мы получаем класс эквивалентности $\phi(x,[w])=\left[\phi^{\prime}, \phi^{\prime \prime}\right]_{\hat{A}_{k}}$ сечений таких, что $\Phi=\hat{A}_{k}(\phi)=\partial_{\square(\phi)}(w)$. По построению оператора $\hat{A}_{k}$ коммутатор

${ }^{5)}$ Из $\ell$-нормальности уравнения $\mathcal{E}_{\mathrm{L}}$ вытекает, что коммутатор $\varphi$ является его симметрией всякий раз, когда скорость $\partial_{\varphi}(w)$ минимальных интегралов лежит в ядре $\left.\operatorname{ker} D_{y}\right|_{\mathcal{E}_{\mathrm{L}}}$ (см. ниже формулу (16)). 
симметрий $\varphi^{\prime}$ и $\varphi^{\prime \prime}$ принадлежит множеству симметрий вида $\square(\phi)$. Это доказывает первое утверждение теоремы.

Вместе с тем таких сечений $\varphi=\square(\phi)$, индуцирующих одну и ту же скорость $\dot{w}=\Phi$, вообще говоря, может оказаться много. Поскольку $\operatorname{ker} \square \subseteq \operatorname{ker} \hat{A}_{k}$, то в принципе класс эквивалентности $\left[\phi^{\prime}, \phi^{\prime \prime}\right]_{\hat{A}_{k}}$ может содержать элементы, которые не принадлежат множеству $\left[\phi^{\prime}, \phi^{\prime \prime}\right] \square$.

Продемонстрируем, что все представители класса эквивалентности $\left[\phi^{\prime}, \phi^{\prime \prime}\right]_{\hat{A}_{k}}$ определяют одну и ту же симметрию $\varphi$ уравнения $\mathcal{E}_{\mathrm{L}}$, и, следовательно, именно симметрия $\varphi=\square(\phi)$ и есть коммутатор $\left[\square\left(\phi^{\prime}\right), \square\left(\phi^{\prime \prime}\right)\right]$ двух исходных симметрий, поскольку образ множества сечений $\phi$ при отображении $\square$ обязан ее содержать. Нам достаточно доказать единственность тривиального решения $\varphi$ для линейного однородного уравнения $\ell_{w}^{(u)}(\varphi)=\left(\ell_{w[\mathfrak{m}]}^{(\mathfrak{m})} \circ \ell_{\mathfrak{m}}^{(u)}\right)(\varphi)=0$.

Имеется три способа получить нулевую скорость $\Phi(x,[w]) \equiv 0$ интегралов $w[\mathfrak{m}[u]]$ вдоль симметрии $\varphi \in \operatorname{sym} \mathcal{E}_{\mathrm{L}}$. Первая возможность - если бы интегралы были дифференциально зависимы - исключается предположением теоремы. Вторая возможность - промежуточное уравнение $\kappa \cdot D_{x}(\varphi)=0$, det $\kappa \neq 0$, может иметь нетривиальные решения, только если какой-то из сдвигов $\varphi=$ const окажется симметрией уравнения $\mathcal{E}_{\mathrm{L}}$. Однако в этом случае определяющее уравнение

$$
\left(D_{x} D_{y}-f^{\prime}(\boldsymbol{u} ; x, y)\right)(\text { const }) \doteq 0,
$$

выполненное для систем $\mathcal{E}_{\mathrm{L}}$, означало бы линейную зависимость между дифференциалами правых частей входящих в нее уравнений, что противоречит другому исходному предположению.

Следовательно, доказательство второго утверждения сводится к задаче единственности нулевого решения $\psi=0$ линейного однородного уравнения $\ell_{w[\mathfrak{m}]}^{(\mathfrak{m})}(\psi)=0$. Поскольку система $\mathcal{E}_{\mathrm{L}}$ - единственный набор ${ }^{6)}$ дифференциальных соотношений, налагаемых на сечения $u=s(x, y) \in \Gamma(\pi)$, нуль в правой части уравнения $\ell_{w}^{(\mathfrak{m})}(\psi)=0$ достигается тождественно по [m] (иначе систему $\mathcal{E}_{\mathrm{L}}$ потребовалось бы переопределить).

Остаются две ситуации, когда скорость $\psi$ импульсов $\mathfrak{m}=-\kappa u_{x} / 2$ обращает величину $\dot{w}=\Phi$ в нуль. Одна из причин очевидна: достаточно использовать само уравнение $\mathcal{E}_{\mathrm{L}}=\left\{\boldsymbol{u}_{x y}=f\right\}$. В самом деле, если "временем" вдоль потока $\dot{\mathfrak{m}}=\psi$ служит переменная $y$, a $\mathfrak{m}_{y}=-\delta H_{\mathrm{L}} / \delta u$, то имеем $\partial_{\mathfrak{m}_{y}}^{(\mathfrak{m})}(w)=D_{y}(w[\mathfrak{m}]) \doteq 0$ на $\mathcal{E}_{\mathrm{L}}$. Однако присутствие переменных $\boldsymbol{u}$ в списке аргументов функции $f$ исключает из дальнейшего рассмотрения такие решения $\psi$.

Без ограничения общности предположим, что интеграл $w^{r}[\mathfrak{m}]$ имеет наивысший дифференциальный порядок: $d(r) \geqslant d(i)$ при всех $i<r$. Вычислим скорости неминимальных интегралов $\left(w^{\prime}\right)^{i}:=D_{x}^{d(r)-d(i)}\left(w^{i}\right)$. Используя перестановочность $\left[D_{x}, \partial_{\psi}^{(\mathfrak{m})}\right]=0$ эволюционных дифференцирований с полными производными, из тождеств $\partial_{\psi}^{(\mathfrak{m})}\left(w^{1}\right)=\cdots=\partial_{\psi}^{(\mathfrak{m})}\left(w^{r}\right)=0$ получим, что $\partial_{\psi}^{(\mathfrak{m})}\left(w^{\prime}\right)=0$. Видно, что линеаризация новых интегралов имеет вид $\ell_{w^{\prime}}^{(\mathfrak{m})}=\Lambda \cdot D_{x}^{d(r)}+O(d(r)-1)$, причем

6) Гиперболическая система $\mathcal{E}_{\mathrm{L}}$ формально интегрируема [12]: ее бесконечное продолжение $\mathcal{E}_{\mathrm{L}}^{\infty}$ существует и имеется эпиморфизм $\mathcal{E}_{\mathrm{L}}^{\infty} \rightarrow \mathcal{E}_{\mathrm{L}}$. 
матрица $\Lambda$ обратима по исходному предположению теоремы. Умножая новое уравнение $\ell_{w^{\prime}}^{(\mathfrak{m})}(\psi)=0$ на $\Lambda^{-1}$, мы по индукции доказываем, что $\psi(x,[\mathfrak{m}])$ не зависит от $[\mathfrak{m}]$, и поэтому $\psi=\psi(x)$. Следовательно, все допустимые сечения $\varphi$, удовлетворяющие промежуточному уравнению $\psi(x)=-\kappa D_{x}(\varphi) / 2$, также зависят лишь от $x: \varphi=\varphi(x)$. Но ни одно из них, кроме нуля, не может быть симметрией гиперболической системы $u_{x y}-f(\boldsymbol{u} ; x, y)=0$ вследствие невырожденности ${ }^{7)}$, поскольку $\operatorname{det} f^{\prime}(\boldsymbol{u}) \neq 0$. "Симметрия" $\varphi(x)$ в наших обозначениях должна удовлетворять определяющему уравнению $\left(D_{x} \circ D_{y}-f^{\prime}(\boldsymbol{u})\right) \varphi(x) \doteq 0$ в силу $\mathcal{E}_{\mathrm{L}}$. Первое слагаемое обращается в нуль, так как $D_{y}(x) \equiv 0$. Значит, $f^{\prime}(u) \cdot \varphi(x)=0$, однако матрица линеаризации $f^{\prime}(\boldsymbol{u})$ обратима. Итак, $\varphi(x) \equiv 0$. Проделанное рассуждение завершает доказательство.

ЗАмЕчАниЕ 4. В приведенном выше доказательстве мы пришли к линейному обыкновенному дифференциальному уравнению $\ell_{w}^{(\mathfrak{m})}(\psi(x))=0$, выполненному одновременно для всех сечений $s \in \Gamma(\pi)$, хотя ненулевые решения $\psi(x)$ и не вносят вклада в алгебру симметрий. Существование нетривиальных решений возможно, прежде всего, если существует оператор $\nabla$ в полных производных такой, что $\ell_{w}^{(\mathfrak{m})} \circ \nabla=0$. (К примеру, тождество $\left(\begin{array}{cc}D_{x} & 1 \\ 0 & 0\end{array}\right)\left(\begin{array}{c}1 \\ -D_{x}\end{array}\right)(h(x)) \equiv 0$ выполнено для всех $h(x)$.) Чтобы избежать этого, необходимо потребовать невырожденности линеаризации интегралов:

$$
\ell_{w[\mathfrak{m}]}^{(\mathfrak{m})} \circ \nabla=0 \Longrightarrow \nabla=0 .
$$

В сопряженной форме, $\nabla^{*} \circ \square=0 \Rightarrow \nabla^{*}=0$, уравнение (12) выражает отсутствие линейных дифференциальных соотношений ${ }^{8)}$ между компонентами симметрий $\varphi=$ $\square(\phi(x,[w]))$.

Указанное свойство двойственно к невырожденности $\nabla \circ \ell_{w}^{(\mathfrak{m})}=0 \Rightarrow \nabla=0$, проистекающей из дифференциально-функциональной независимости $\Phi(x,[w])=0 \Rightarrow$ $\Phi \equiv 0$, если положить $\nabla=\ell_{\Phi}^{w}($ см. сноску 4$)$.

Наконец, пусть сечение $s(x, y) \in \operatorname{Sol} \mathcal{E}_{\mathrm{L}}-$ некоторое решение интегрируемой по Дарбу системы $\mathcal{E}_{\mathrm{L}}$ лиувиллева типа. Взяв ограничение $\mathcal{L}^{s}=\left.\ell_{w}^{(\mathfrak{m})}\right|_{j_{\infty}(s)}$ оператора линеаризации на струю сечения $s$, мы приходим к обыкновенному дифференциальному уравнению $\mathcal{L}^{s}(\psi(x))=0$. Для каждого $s$ линейное пространство $\mathcal{O}(s)$ его решений конечномерно. (В частности, эта размерность равна сумме показателей полупростой комплексной алгебры Ли, если $\mathcal{E}_{\mathrm{L}}-$ ассоциированная с ней двумерная цепочка Тоды.) Следовательно, требование

$$
\bigcap_{s \in \operatorname{Sol} \mathcal{E}_{\mathrm{L}} \subset \Gamma(\pi)} \mathcal{O}(s)=\{0\}
$$

в сочетании с (12) позволяет устранить избыточную свободу в выборе решений $\psi(x)$ уравнения $\ell_{w}^{(\mathfrak{m})}(\psi)=0$.

\footnotetext{
7)Это утверждение - непосредственное, проверяемое в каждой точке $x$ обобщение того факта (см. выше), что $\varphi=$ const $\neq 0$ не является симметрией уравнения $\mathcal{E}_{\mathrm{L}}$.

8) Отсюда следует, что невырожденность - уравнение (12) - аналогична понятию $\ell$-нормальности дифференциальных уравнений, возникающему при анализе их формальной интегрируемости (см. раздел 4).
} 
Теорема 2 была иллюстрирована на примерах полупростых комплексных алгебр Ли ранга 2 в работе [16], где выписаны гамильтоновы операторы $\hat{A}_{1}$ и $\hat{A}_{k}$ для соответствующих иерархий Дринфельда-Соколова [11], а затем вычислены коммутационные соотношения в $\operatorname{sym} \mathcal{E}_{\mathrm{L}}$ для двумерных цепочек Тоды $\boldsymbol{u}_{x y}=e^{K \boldsymbol{u}}$.

Пример 3 (модифицированное уравнение Каупа-Буссинеска). Рассмотрим лагранжево расширение скалярного уравнения Лиувилля [15]:

$$
A_{x y}=-\frac{1}{8} A e^{-B / 4}, \quad B_{x y}=\frac{1}{2} e^{-B / 4} .
$$

Обозначим импульсы как $a=B_{x} / 2$ и $b=A_{x} / 2$. Минимальные интегралы системы (13) суть $w_{1}=-a^{2} / 4-a_{x}$ и $w_{2}=a b+2 b_{x}$, так что $D_{y}\left(w_{i}\right) \doteq 0, i=1,2$, в силу (13). Поэтому оператор

$$
\square=\left(\ell_{w_{1}, w_{2}}^{(a, b)}\right)^{*}=\left(\begin{array}{cc}
-\frac{1}{4} B_{x}+D_{x} & \frac{1}{2} A_{x} \\
0 & \frac{1}{2} B_{x}-2 D_{x}
\end{array}\right)
$$

порождает нётеровы (см. (4)) симметрии уравнения (13). Скобка $\{\{\cdot, \cdot\}\}_{\square}$, индуцированная на области определения оператора $\square$, имеет вид

$$
\{\{\boldsymbol{\psi}, \boldsymbol{\chi}\}\}_{\square}=\frac{1}{2}\left(\begin{array}{c}
\psi_{x}^{2} \chi^{1}-\psi^{1} \chi_{x}^{2}+\psi_{x}^{1} \chi^{2}-\psi^{2} \chi_{x}^{1} \\
\psi_{x}^{2} \chi^{2}-\psi^{2} \chi_{x}^{2}
\end{array}\right),
$$

где $\boldsymbol{\psi}={ }^{t}\left(\psi^{1}, \psi^{2}\right)$ и $\boldsymbol{\chi}={ }^{t}\left(\chi^{1}, \chi^{2}\right)$.

Рассмотрим симметрию уравнения (13):

$$
A_{t}=\frac{1}{2} A_{x} A_{x x}+\frac{1}{2}\left(\frac{1}{4} A_{x}^{2}-1\right) B_{x}, \quad B_{t}=-2 A_{x x x}+\frac{1}{8} A_{x} B_{x}^{2}-\frac{1}{2} A_{x} B_{x x} .
$$

Выберем эквивалентную пару интегралов $u=w_{2}, v=w_{1}+w_{2}^{2} / 4$. Эволюция переменных $u$ и $v$ вдоль (14) задана уравнениями

$$
u_{t}=u u_{x}+v_{x}, \quad v_{t}=(u v)_{x}+u_{x x x} .
$$

Мы получили систему Каупа-Буссинеска, а (14) - потенциальное дважды модифицированное уравнение Каупа-Буссинеска (см. [22]). Правая часть интегрируемой системы (14) принадлежит образу сопряженной линеаризации $\widetilde{\square}=\left(\ell_{(u, v)}^{(a, b)}\right)^{*}$. Оператор $\tilde{\square}$ факторизует третью гамильтонову структуру $\hat{A}_{3}^{\mathrm{KB}}=\tilde{\square}^{*} \circ\left(\ell_{(a, b)}^{(A, B)}\right)^{*} \circ \tilde{\square}$ для уравнений (15); итак, $k=3$ и

$$
\hat{A}_{3}^{\mathrm{KB}}=\left(\begin{array}{cc}
u D_{x}+\frac{1}{2} u_{x} & D_{x}^{3}+\left(\frac{1}{4} u^{2}+v\right) D_{x}+\frac{1}{4}\left(u^{2}+2 v\right)_{x} \\
D_{x}^{3}+\left(\frac{1}{4} u^{2}+v\right) D_{x}+\frac{1}{2} v_{x} & u D_{x}^{3}+\frac{3}{2} u_{x} D_{x}^{2}+\left(\frac{3}{2} u_{x x}+u v\right) D_{x}+ \\
+\frac{1}{2} u_{x x x}+\frac{1}{2}(u v)_{x}
\end{array}\right) .
$$


Согласно теореме 2 скобка $\{\{\cdot, \cdot\}\}_{\tilde{\square}}$ равна скобке $\{\{\cdot, \cdot\}\}_{\hat{A}_{3}^{\text {кв}}}$, заданной формулой (10). Тем самым имеем

$$
\{\{\boldsymbol{\psi}, \boldsymbol{\chi}\}\}_{\widetilde{\square}}=\{\{\boldsymbol{\psi}, \boldsymbol{\chi}\}\}_{\hat{A}_{3}^{\mathrm{KB}}}=\left(\begin{array}{l}
\boldsymbol{\psi} \cdot \nabla_{1}(\boldsymbol{\chi})-\nabla_{1}(\boldsymbol{\psi}) \cdot \boldsymbol{\chi} \\
\boldsymbol{\psi} \cdot \nabla_{2}(\boldsymbol{\chi})-\nabla_{2}(\boldsymbol{\psi}) \cdot \chi
\end{array}\right),
$$

где

$$
\nabla_{1}=-\frac{1}{2}\left(\begin{array}{cc}
D_{x} & 0 \\
u D_{x} & D_{x}^{3}+v D_{x}
\end{array}\right), \quad \nabla_{2}=-\frac{1}{2}\left(\begin{array}{cc}
0 & D_{x} \\
D_{x} & u D_{x}
\end{array}\right) .
$$

Оператор $\hat{A}_{1}=\left(\begin{array}{cc}0 & D_{x} \\ D_{x} & 0\end{array}\right)$ - первая гамильтонова структура для уравнений (15), обратный к нему оператор $A_{1}=\hat{A}_{1}^{-1}$ факторизует вторую гамильтонову структуру $B_{2}=\widetilde{\square} \circ A_{1} \circ \widetilde{\square}$ уравнения (14).

\section{4. НЕЛАГРАНЖЕВЫ СИСТЕМЫ ЛИУВИЛЛЕВА ТИПА}

Пусть $\mathcal{E}=\{F=0\}$ - система лиувиллева типа, но не обязательно Эйлера-Лагранжа. Предположим, что столбец $\left.w \in \operatorname{ker} D_{y}\right|_{\mathcal{E}}$ состоит из минимальных интегралов уравнения $\mathcal{E}$, а потому $D_{y}(w)=\nabla(F)$ для некоторого оператора $\nabla$. Из структуры систем лиувиллева типа $\mathcal{E}$ ясно, что между входящими в них гиперболическими уравнениями $\left\{F^{i}=0\right\}$ не может существовать дифференциальных связей (сизигий): из предположения $\Delta(F)=0$ следует, что $\Delta=0$. По той же причине системы $\mathcal{E}$ являются $\ell$-нормальными [12], [14]: если $\Delta \circ \ell_{F} \doteq 0$ на $\mathcal{E}$, то также имеем $\Delta=0$. Следовательно, эволюционное векторное поле $\partial_{\varphi}$ будет симметрией системы лиувиллева типа $\mathcal{E}$ в том и только том случае, если оно сохраняет интегралы:

$$
D_{y}\left(\partial_{\varphi}(w)\right)=\partial_{\varphi}(\nabla)(F)+\nabla\left(\partial_{\varphi}(F)\right) \doteq \nabla\left(\ell_{F}(\varphi)\right) \quad \text { на } \quad \mathcal{E}
$$

Рассмотрим операторное уравнение

$$
D_{y} \circ \ell_{w}^{(u)} \doteq \nabla \circ \ell_{F} \quad \text { на } \quad \mathcal{E} .
$$

Если, предположим, удалось бы построить такой оператор $\square$ в полных производных, что

$$
\ell_{w}^{(u)} \circ \square \in \mathcal{C} \operatorname{Diff}\left(\left.\left.\operatorname{ker} D_{y}\right|_{\mathcal{E}} \rightarrow \operatorname{ker} D_{y}\right|_{\mathcal{E}}\right),
$$

то всякому $r$-компонентному набору $\phi(x,[w])$ интегралов (см. (5)) он ставил бы в соответствие симметрию $\varphi=\square(\phi)$ системы $\mathcal{E}$ лиувиллева типа.

В работе [10] содержится алгоритм построения операторов $\square$ - решений уравнения в полных производных

$$
\ell_{w}^{(u)} \circ \square=\mathbf{1}_{m \times m} \cdot D_{x}^{k} \quad \bmod \mathcal{C} \operatorname{Diff}_{<k}\left(\left.\left.\operatorname{ker} D_{y}\right|_{\mathcal{E}} \rightarrow \operatorname{ker} D_{y}\right|_{\mathcal{E}}\right) .
$$

Наиболее примечательно, что обрыв снизу в последовательности коэффициентов при степенях производных в операторах $\square$ следует из существования полного набора интегралов $\left.\bar{w} \in \operatorname{ker} D_{x}\right|_{\mathcal{E}}$ для системы $\mathcal{E}$. Впрочем, минимальные интегралы $w$ приходится "портить" дифференцированием по $x$ подходящее число раз. В результате вместо гамильтонова оператора $\hat{A}_{k}=\ell_{w}^{(u)} \circ \square($ см. (7)) получается правая часть 
уравнения (18). Кроме того, оказывается, что образы построенных в работе [10] операторов не всегда порождают всю $x$-компоненту алгебры Ли $\operatorname{sym} \mathcal{E}$ целиком и, вообще говоря, не замкнуты относительно коммутирования. В заключение приходится признать, что при обратимых заменах $\widetilde{w}[w]$ интегралов нелагранжевых систем лиувиллева типа правила преобразования сечений, принадлежащих области определения операторов $\square$, остаются не определены.

УтВЕРЖДЕНИЕ 2. Если образ решения $\square$ операторного уравнения (17) для системы лиувиллева типа $\mathcal{E}$ замкнут относительно коммутирования, то все коэббициенты скобки $\{\{\cdot, \cdot\}\}_{\square}$, возникающей на его области определения (cм. (9)), лежат в ядре $\left.\operatorname{ker} D_{y}\right|_{\mathcal{E}}$.

ДокАЗАТЕЛЬство. По предположению имеем $\left(D_{y} \circ \ell_{w}^{(u)} \circ \square\right)\left(\left[\phi^{\prime}, \phi^{\prime \prime}\right] \square\right) \doteq 0$ для всех $\phi^{\prime}, \phi^{\prime \prime}(x,[w])$. Распишем это равенство подробнее:

$0 \doteq\left(D_{y} \circ \underline{\ell_{w}^{(u)} \circ \square}\right)\left(\partial_{\square\left(\phi^{\prime}\right)}\left(\phi^{\prime \prime}\right)-\partial_{\square\left(\phi^{\prime \prime}\right)}\left(\phi^{\prime}\right)+\left\{\left\{\phi^{\prime}, \phi^{\prime \prime}\right\}\right\}_{\square}\right) \doteq\left(\ell_{w}^{(u)} \circ \square\right)\left(D_{y}\left(\left\{\left\{\phi^{\prime}, \phi^{\prime \prime}\right\}\right\}_{\square}\right)\right)$,

поскольку подчеркнутая композиция удовлетворяет условию (17). Ясно, что производные $D_{y}\left(\phi^{\prime}\right)$ и $D_{y}\left(\phi^{\prime \prime}\right)$ обращаются в нуль на $\mathcal{E}$ для любых $\phi^{\prime}, \phi^{\prime \prime}$. По тем же причинам не только вся скобка $\left\{\left\{\phi^{\prime}, \phi^{\prime \prime}\right\}\right\}_{\square}$, но и каждый из коэффициентов, стоящих при билинейных слагаемых в ней, лежит в ядре ker $\left.D_{y}\right|_{\mathcal{E}}$.

ПримеР 4. Рассмотрим параметрическое расширение скалярного уравнения Лиувилля:

$$
\mathcal{E}(\varepsilon)=\left\{u_{x y}=e^{2 u} \sqrt{1+4 \varepsilon^{2} u_{x}^{2}}\right\}, \quad \varepsilon \in \mathbb{R} .
$$

Это уравнение является объемлющим для иерархии уравнения, представляющего собой деформацию по Гарднеру потенциального модифицированного уравнения КдФ (см. [15]). Контракция $\mathcal{U}=\mathcal{U}(\varepsilon,[u(\varepsilon)])$ из (19) в нерасширенное уравнение $\mathcal{U}_{x y}=e^{2 \mathcal{U}}$ задана формулой $\mathcal{U}=u+\frac{1}{2} \operatorname{arcsh}\left(2 \varepsilon u_{x}\right)$; если подставить ее в интеграл $w=\mathcal{U}_{x}^{2}-\mathcal{U}_{x x}$ при $\varepsilon=0$, получится интеграл третьего порядка для уравнения (19). Вместе с тем регуляризованный (при $\varepsilon=0$ ) интеграл меньшего (второго) порядка для уравнения (19) имеет вид

$$
w=\frac{1-\sqrt{1+4 \varepsilon^{2} u_{x}^{2}}}{2 \varepsilon^{2}}+\frac{u_{x x}}{\sqrt{1+4 \varepsilon^{2} u_{x}^{2}}} .
$$

Второй интеграл для уравнения (19) есть $\bar{w}=u_{y y}-u_{y}^{2}-\left.\varepsilon^{2} e^{4 u} \in \operatorname{ker} D_{x}\right|_{\mathcal{E}(\varepsilon)}$. Операторы

$$
\begin{gathered}
\square=u_{y}+\frac{1}{2} D_{y}, \\
\square=\frac{1}{2}\left(1+4 \varepsilon^{2} u_{x}^{2}-2 \varepsilon^{2} u_{x x}\right) D_{x}+u_{x}+4 \varepsilon^{2} u_{x}^{3}-2 \varepsilon^{2} u_{x x x}+\frac{12 \varepsilon^{4} u_{x} u_{x x}^{2}}{1+4 \varepsilon^{2} u_{x}^{2}}
\end{gathered}
$$

отображают интегралы уравнения (19) в его симметрии, $\varphi=\square(\phi(x,[w]))$ и $\bar{\varphi}=$ $\bar{\square}(\bar{\phi}(y,[\bar{w}]))$. 
Образ каждого из операторов $\square$ и $\square-$ подалгебра Ли в $\operatorname{sym} \mathcal{E}(\varepsilon)$. Скобка $\{\{p, q\}\}_{\square}=p_{y} q-p q_{y}$ для $\bar{\square}$ записывается в привычном виде [3], [9]. А скобка, индуцированная на области определения оператора $\square$, имеет следующий вид: для любых аргументов $p, q$ получаем

$$
\begin{aligned}
\{\{p, q\}\}_{\square}=\varepsilon^{2}\left(p_{x x} q_{x}-p_{x} q_{x x}\right)-2 \varepsilon^{2}\left(p_{x x x} q-p q_{x x x}\right)- \\
\quad-12 \varepsilon^{4}\left(8 \varepsilon^{2} u_{x}^{3} u_{x x}-4 \varepsilon^{2} u_{x}^{2} u_{x x x}+4 \varepsilon^{2} u_{x} u_{x x}^{2}+2 u_{x} u_{x x}-u_{x x x}\right) \times \\
\quad \times\left(1+8 \varepsilon^{2} u_{x}^{2}+16 \varepsilon^{4} u_{x}^{4}-2 \varepsilon^{2} u_{x x}-8 \varepsilon^{4} u_{x}^{2} u_{x x}\right)^{-1}\left(p_{x x} q-p q_{x x}\right)+ \\
\quad+\left(\underline{1}+288 \varepsilon^{4} u_{x}^{4}-288 \varepsilon^{4} u_{x}^{2} u_{x x}+28 \varepsilon^{2} u_{x}^{2}-16 \varepsilon^{2} u_{x x}-288 \varepsilon^{6} u_{x} u_{x x} u_{x x x}-\right. \\
\quad-96 \varepsilon^{6} u_{x x}^{3}+3072 \varepsilon^{10} u_{x}^{10}+24 \varepsilon^{6} u_{x x x}^{2}+24 \varepsilon^{4} u_{4 x}+1408 \varepsilon^{6} u_{x}^{6}+3328 \varepsilon^{8} u_{x}^{8}- \\
\quad-768 \varepsilon^{10} u_{4 x} u_{x x} u_{x}^{4}-384 \varepsilon^{8} u_{4 x} u_{x}^{2} u_{x x}-2304 \varepsilon^{8} u_{x}^{3} u_{x x} u_{x x x}+384 \varepsilon^{8} u_{x x}^{2} u_{x} u_{x x x}- \\
\quad-4608 \varepsilon^{10} u_{x}^{5} u_{x x} u_{x x x}+16 \varepsilon^{4} u_{x x}^{2}-5632 \varepsilon^{8} u_{x}^{6} u_{x x}-1920 \varepsilon^{6} u_{x x} u_{x}^{4}+3328 \varepsilon^{8} u_{x}^{4} u_{x x}^{2}+ \\
\quad+512 \varepsilon^{6} u_{x x}^{2} u_{x}^{2}+384 \varepsilon^{10} u_{x}^{4} u_{x x x}^{2}-960 \varepsilon^{10} u_{x x}^{4} u_{x}^{2}-48 \varepsilon^{4} u_{x} u_{x x x}-3072 \varepsilon^{10} u_{x}^{7} u_{x x x}+ \\
\quad+3072 \varepsilon^{10} u_{x x}^{3} u_{x}^{4}-2304 \varepsilon^{8} u_{x}^{5} u_{x x x}-576 \varepsilon^{6} u_{x}^{3} u_{x x x}+288 \varepsilon^{6} u_{4 x} u_{x}^{2}+384 \varepsilon^{8} u_{x}^{2} u_{x x}^{3}+ \\
+6144 \varepsilon^{10} u_{x x}^{2} u_{x}^{6}-6144 \varepsilon^{10} u_{x x} u_{x}^{8}+1152 \varepsilon^{8} u_{4 x} u_{x}^{4}+1536 \varepsilon^{10} u_{4 x} u_{x}^{6}+192 \varepsilon^{8} u_{x x x}^{2} u_{x}^{2}+ \\
\left.\quad+240 \varepsilon^{8} u_{x x}^{4}+1536 \varepsilon^{10} u_{x x}^{2} u_{x}^{3} u_{x x x}-48 \varepsilon^{6} u_{4 x} u_{x x}\right) \times \\
\quad \times\left(1+96 \varepsilon^{4} u_{x}^{4}+256 \varepsilon^{6} u_{x}^{6}+256 \varepsilon^{8} u_{x}^{8}+4 \varepsilon^{4} u_{x x}^{2}-48 \varepsilon^{4} u_{x}^{2} u_{x x}+32 \varepsilon^{6} u_{x x}^{2} u_{x}^{2}-\right. \\
\left.\quad-4 \varepsilon^{2} u_{x x}-256 \varepsilon^{8} u_{x}^{6} u_{x x}+64 \varepsilon^{8} u_{x}^{4} u_{x x}^{2}-192 \varepsilon^{6} u_{x x} u_{x}^{4}+16 \varepsilon^{2} u_{x}^{2}\right)^{-1}\left(p_{x} q-p q_{x}\right) .
\end{aligned}
$$

Две подчеркнутые единицы соответствуют скобке $p_{x} q-p q_{x}$ на области определения оператора $\square=\mathcal{U}_{x}+D_{x} / 2$, который задает симметрии уравнения Лиувилля $\mathcal{U}_{x y}=e^{2 \mathcal{U}}$ при $\varepsilon=0$. В соответствии с утверждением 2 непостоянные коэффициенты при билинейных слагаемых $p_{x x} q-p q_{x x}$ и $p_{x} q-p q_{x}$ в $\{\{p, q\}\}_{\square}$ принадлежат ядру $\left.\operatorname{ker} D_{y}\right|_{\mathcal{E}(\varepsilon)}$. Примечательно следующее: поскольку все конструкции (19)-(21) содержат формальные степенные ряды $u(\varepsilon)$ по $\varepsilon$, той же природы оказываются и эти две рациональные функции. Попытка выразить их через $w$ и производные $w$ по $x$ приводит к формальным рядам с неограниченным ростом дифференциальных порядков в коэффициентах.

\section{5. ОБСУЖДЕНИЕ}

Матричные операторы $\square=\left(\square^{i, j}, 1 \leqslant i \leqslant m, 1 \leqslant j \leqslant r\right)$, заданные формулой (3), являются (применительно к геометрии расслоений бесконечных струй) обобщениями тензоров типа $(2,0)$. Мы дали определение этих операторов, учитывая наличие двух не связанных между собой групп дифференциальных подстановок на координатах в их области определения и, соответственно, в образе. С другой стороны, операторы $\square$ для систем лиувиллева типа $\mathcal{E}_{\mathrm{L}}$ обобщают теорию гамильтоновых структур следующим образом: они отображают вариационные ковекторы для одного уравнения (напомним, что $\operatorname{sym} \mathcal{E}_{\varnothing} \supset \mathfrak{A}$ ) в симметрии другой системы $\mathcal{E}_{\mathrm{L}}$ (такой, что $\left.\operatorname{sym} \mathcal{E}_{\mathrm{L}} \supset \mathfrak{B}\right)$.

2 Теоретическая и математическая физика, т. 162, № 2, 2010 г. 
В отличие от работ [8], [10], здесь мы не пытаемся решить уравнение (17) относительно $\square$. Напротив, мы определили операторы (3), пользуясь геометрическими рассуждениями. Благодаря этому мы, во-первых, получили гамильтоновы операторы $\hat{A}_{k}=\ell_{w}^{(u)} \circ \square$ для иерархий типа КдФ на лагранжевых системах лиувиллева типа [9], [11] и, во-вторых, доказали, что образы операторов $\square$ инволютивны (см. [23]). Иными словами, был описан конструктивный алгоритм, назначение которого - порождение вполне интегрируемых уравнений.

Формулы (3) и (7) позволяют предсказать дифференциальный порядок оператора $\hat{A}_{k}$. Оценки порядков интегралов $w$ для двумерных цепочек Тоды, ассоциированных с полупростыми комплексными алгебрами Ли $\mathfrak{g}$, известны из работы [2] (см. пример 1), в иных формулировках они содержатся в работах [4], [16]. Верхняя граница - чи́сла $\operatorname{ord}_{x} w^{i}-1$ не превышают показателей алгебры Ли $\mathfrak{g}$ - доказывается непосредственной проверкой (с использованием полиномов Шура) соотношений Cерра $\left(\operatorname{ad} Y_{i}\right)^{-K_{j}^{i}+1}\left(Y_{j}\right)=0, i \neq j$, для образующих

$$
Y_{i}=\sum_{k \geqslant 0} \exp \left(-\sum_{j=1}^{m} K_{j}^{i} u^{j}\right) \cdot D_{x}^{k}\left(\exp \left(\sum_{j^{\prime}=1}^{m} K_{j^{\prime}}^{i} u^{j^{\prime}}\right)\right) \cdot \frac{\partial}{\partial u_{k+1}^{i}}
$$

характеристических алгебр Ли (см. [1], [2], [7], а также [16]) и применением теоремы Фробениуса. Здесь важно то, что векторные поля $Y_{i}$ в точности совпадают с образующими Шевалле $\mathfrak{f}_{i}$ полупростой алгебры Ли g. Эта же оценка снизу следует из отсутствия соотношений, отличных от соотношений Серра, между образующими $Y_{i}$. Для систем корней $\mathrm{A}_{n}$ и $\mathrm{D}_{n}$ данный факт был установлен в работе [2] явным перечислением линейно независимых ненулевых итерированных коммутаторов.

Благодарности. Авторы благодарны Б.А. Дубровину, И.С. Красильщику, М. А. Нестеренко, П. Олверу, В.В.Соколову и Е. В. Ферапонтову за обсуждения и высказанные замечания. Авторы признательны рецензентам за полезные советы. А. В. Киселев благодарит организационный комитет Международного совещания "Nonlinear Physics: Theory and Experiment V" за поддержку и гостеприимство. Настоящая работа была частично поддержана Европейским Союзом (грант FP6 Marie Curie RTN ENIGMA - контракт MRTN-CT-2004-5652), а также European Science Foundation Program MISGAM и грантами NWO B61-609 и VENI 639.031.623. Часть данного исследования была выполнена во время стажировок А. В. Киселева в IHÉS, SISSA и CRM (Монреаль), всем этим организациям благодарность за финансовую поддержку.

\section{Список литературы}

[1] А. Н. Лезнов, В. Г. Смирнов, А. Б. Шабат, ТМФ, 51:1 (1982), 10-21.

[2] А.Б. Шабат, Р. И. Ямилов, Экспоненциальные системы типа I и матрицы Картана, Препринт Башкир. отд. АН СССР, Уфа, 1981.

[3] А. В. Жибер, В. В. Соколов, УМН, 56:1(337) (2001), 63-106.

[4] А. М. Гурьева, А. В. Жибер, ТМФ, 138:3 (2004), 401-421.

[5] A. N. Leznov, M. V. Saveliev, Lett. Math. Phys., 3:6 (1979), 489-494.

[6] А. Г. Мешков, ТМФ, 63:3 (1985), 323-332.

[7] A. B. Shabat, Phys. Lett. A, 200:2 (1995), 121-133. 
[8] Д. К. Демской, С. Я. Старцев, Фундамент. и прикл. матем., 10:1 (2004), 29-37.

[9] А.В. Киселев, ТМФ, 144:1 (2005), 83-93.

[10] В. В. Соколов, С. Я. Старцев, ТМФ, 155:2 (2008), 344-355.

[11] В. Г. Дринфельд, В. В. Соколов, “Алгебры Ли и уравнения типа Кортевега-де Фриза”, Итоги науки и техники. Сер. Современные проблемы математики. Нов. достиж., 24, ред. Р. В. Гамкрелидзе, ВИНИТИ, М., 1984, 81-180.

[12] А. М. Виноградов, И. С. Красильщик (ред.), Симметрии и законы сохранения уравнений математической физики, Факториал, М., 1997.

[13] П. Олвер, Приложения групп Ли к дифберенииалъным уравнениям, Мир, М., 1989.

[14] J. Krasil'shchik, A. Verbovetsky, Homological methods in equations of mathematical physics, arXiv: math. DG/9808130.

[15] А.В. Киселев, ТМФ, 152:1 (2007), 101-117.

[16] A. V. Kiselev, J. W. van de Leur, A geometric derivation of KdV-type hierarchies from root systems, Proc. 4-th Int. Workshop "Group Analysis of Differential Equations and Integrable Systems" (Protaras, Cyprus, 2008), 2009, 87-106; arXiv: 0901.4866.

[17] N.H. Ibragimov, A.V. Aksenov, V.A. Baikov, V.A. Chugunov, R. K. Gazizov, A. G. Meshkov, CRC Handbook of Lie Group Analysis of Differential Equations. Vol. 2. Applications in Engineering and Physical Sciences, ed. N. H. Ibragimov, Boca Raton, FL, CRC Press, 1995.

[18] С. Я. Старцев, Фундамент. и прикл. матем., 12:7 (2006), 251-262.

[19] В. В. Соколов, УМН, 43:5(263) (1988), 133-163.

[20] С. Я. Старцев, ТМФ, 116:3 (1998), 336-348.

[21] B. A. Kupershmidt, G. Wilson, Invent. Math., 62:3 (1980), 403-436.

[22] M. Pavlov, J. Nonlinear Math. Phys., 9, suppl. 1 (2002), 173-191.

[23] A. V. Kiselev, J. W. van de Leur, Involutive distributions of operator-valued evolutionary vector fields. II, arXiv: 0904.1555.

Поступила в редакцию 26.02.2009, после доработки 18.05.2009 